\title{
High Resolution X-ray Spectroscopy: Is It Interesting? Is It Possible?
}

\author{
Webster Cash \\ University of Colorado, Boulder, CO 80309-0391 USA
}

\begin{abstract}
The diffraction grating spectra from Chandra and XMM-Newton have given the astronomical community a huge step forward in x-ray spectroscopy of celestial sources. They have proven the scientific richness of the field. But the spectra have resolution of only 300 to 1000 - low by the standards of the visible and the ultraviolet. We discuss some of the exciting new science that can be addressed if spectral resolution of up to 10,000 (or more) can be achieved in the x-ray. We then show how practical, high efficiency, high resolution $\mathrm{x}$-ray spectrographs can be built for high throughput missions like Constellation-X and XEUS.
\end{abstract}

After nearly two decades of development, the Chandra Observatory and XMM-Newton were successfully launched. Each features both imaging and spectroscopy modes in the x-ray band from 0.1 to $8 \mathrm{keV}$. Each has been spectacularly successful. The images from Chandra, featuring unprecedented subarcsecond resolution coupled with high collecting area, have revolutionized our view of the $\mathrm{x}$-ray universe. But, until these two observatories were launched, professional quality x-ray spectra of celestial sources simply did not exist. All prior observatories with spectroscopic capability had very low resolution, typically below 30 . Huge advances in our understanding of $x$-ray sources were made using this low resolution, but most of the spectral information carrying key clues to the nature of the sources was irretrievably blurred.

Chandra and XMM-Newton, with resolution of 300 up to 1500 and collecting areas in the 50 to 300 square centimeter range, reveal the true complexity of the spectra for the first time. The $\mathrm{x}$-ray region, like the ultraviolet, is rich in atomic diagnostics that give basic information about the state of the emitting source and other material along the line of sight. After three years of such data, we are only scratching the surface of what is to come.

But what is needed to continue for the future? Chandra and XMM are limited by their size so more collecting area is needed. Planning is already underway for the next generation of x-ray spectrographs, most prominently for Constellation-X in the USA and XEUS in Europe. In the case of Constellation$\mathrm{X}$, the goal is to improve the collecting area of the spectrograph by an order of magnitude over XMM-Newton. XEUS, which will follow Con-X into orbit, will provide another order of magnitude yet again. These future missions will be able to reach truly faint $\mathrm{x}$-ray sources.

In the visible portion of the spectrum astronomers value spectral resolution, but usually not at the expense of collecting area. As astronomy matured over the last century it became clear that spectral resolution can, if it comes at the expense of sensitivity, hinder an observation. This brings to mind the question for x-ray spectroscopy: Is high resolution interesting and if so, is it possible? 


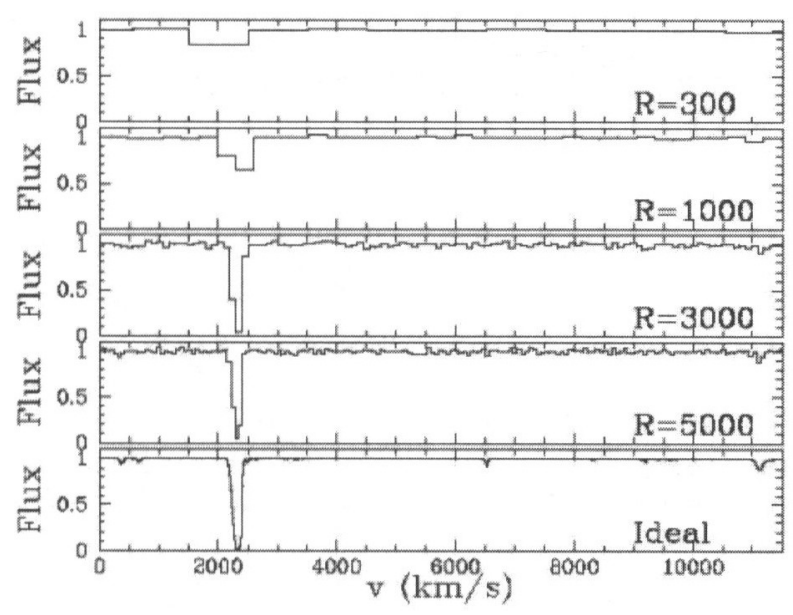

Figure 1. Simulated spectrum of the OVII line from the intergalactic medium smoothed to resolutions of $300,1000,3000$ and 5000 . It is clear that resolution of 1000 is inadequate for this problem, but 3000 appears to be sufficient. (Courtesy N. Gnedin)

What is high resolution in the x-ray band? Anything of resolution higher than is available now is usually considered to be high, meaning that as we push beyond the $\mathrm{R}=1000$ limit of Chandra, we are considering high resolution.

One simple way to look at high resolution is to consider the case of absorption lines. Surely the next generation of observatories, with its huge collecting area, should be designed to resolve absorption lines from gas along the line of sight in the manner that ultraviolet astronomers have been doing for four decades. N. Gnedin has kindly provided me with a simulation of such an absorption line as shown in Figure 1. It simulates the O VII absorption line in the $\mathrm{x}$-ray along a line of sight through the intergalactic medium. Since this observation is of fundamental importance to understanding the intergalactic medium and the formation of structure in the early universe, it is therefore of major importance to the future of x-ray astronomy (Hellston, Gnedin, \& Miralda-Escude 1998). The figure shows simulated observations across a range of resolutions from 300 to 5000 . It is clear that even resolution of 1000 is not sufficient to perform this important diagnostic: 3000 looks sufficient, and 5000 is definitely good enough.

Next one must address the feasibility of achieving these kinds of resolutions in a practical and affordable way. Unfortunately, the approach used by Chandra is unsuitable to Constellation-X and XEUS because these missions will feature large aperture, densely packed arrays of thin mirrors with spatial resolution poorer than Chandra's. XMM uses an array of reflection gratings to increase dispersion and thereby achieves spectral resolution of 300 . Constellation- $\mathrm{X}$ is baselined on a similar configuration (Kahn et al. 1999).

Recently, at the University of Colorado, we have been studying an alternative. It again features reflection gratings, but used in the off-plane mount. This concept, as a means for achieving high spectral resolution in the $\mathrm{x}$-ray with 


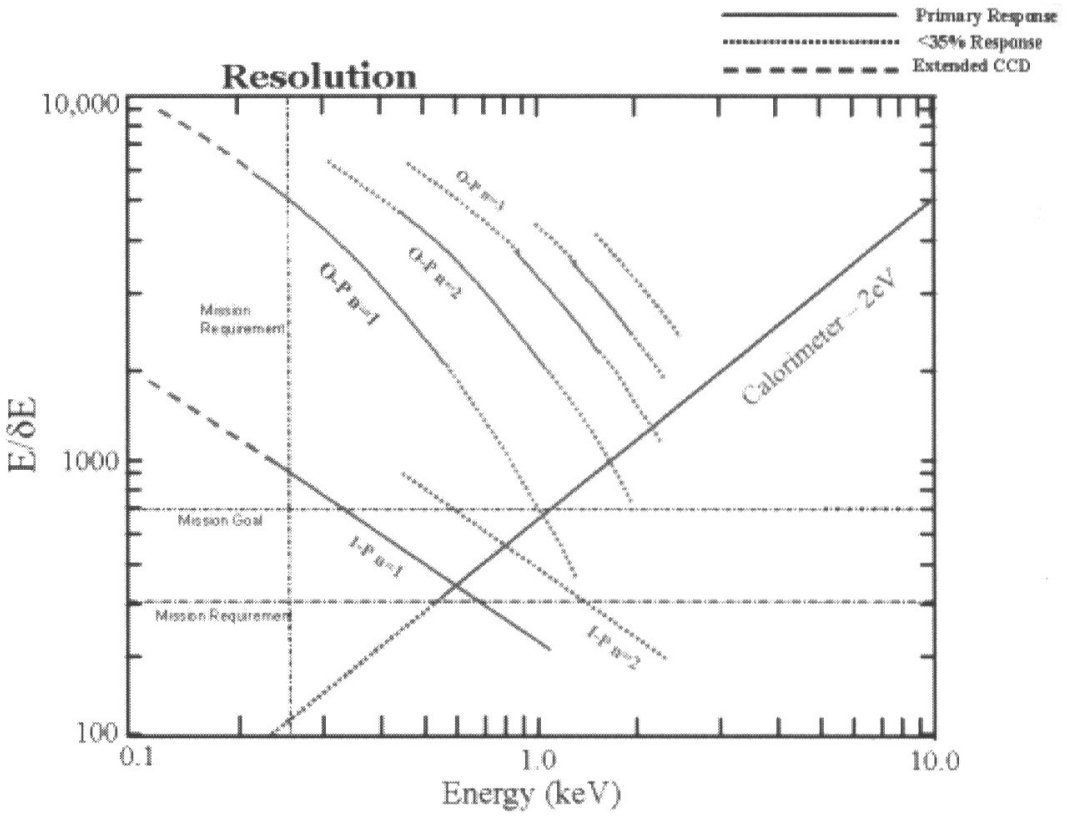

Figure 2. The diagram shows the resolution of the Constellation-X experiment as a function of spectral energy. The straight line that rises to the right is the calorimeter experiment that is optimized for work with the astrophysically significant $\mathrm{Fe} \mathrm{K}$ line at $6 \mathrm{keV}$. The straight line in the lower left represents the expected performance of an in-plane reflection grating spectrograph. The curved lines show the potential for improvement represented by the off-plane mount.

modest quality telescopes, was described by Cash (1991). Development is now being supported by NASA through the Constellation-X project, and so far the results look good. Figure 2 shows the eventual possible performance of an offplane mount grating array on Con-X (McEntaffer, Cash, \& Shipley 2002). This approach has the potential to reach the really compelling resolution of 3000 .

In summary, high resolution x-ray spectroscopy is very, very interesting, and appears to be achievable within the constraints of the next generation of x-ray observatories. This paper was supported by NASA grant NAG5-11850.

\section{References}

Cash, W. 1991, Appl. Opt., 30, 1749

Hellsten, U., Gnedin, N. Y., \& Miralda-Escude, J. 1998, ApJ, 509, 56

Kahn, S. K., et al. 1999, Proc. Soc. Photo-Opt. Instr. Eng., 3765, 94

McEntaffer, R., Cash, W., \& Shipley, A. 2002, Proc. Soc. Photo-Opt. Instr. Eng., 4851,549 